Check for updates

Cite this: RSC Adv., 2019, 9, 7228

Received 27th November 2018 Accepted 23rd January 2019

DOI: $10.1039 / c 8 r a 09740 b$

rsc.li/rsc-advances

\section{Silver-modified porous polystyrene sulfonate derived from Pickering high internal phase emulsions for capturing lithium-ion}

\begin{abstract}
Xiaojing Wang, ${ }^{a}$ Xueping Chen, ${ }^{b}$ Yinxian Peng*a and Jianming Pan (D) *b
Adsorption separation based on porous polystyrene sulfonate is an important method of extracting lithium ion $\left(\mathrm{Li}^{+}\right)$. In this work, silver-modified porous polystyrene sulfonate (PHIPEs-SS-Ag) derived from Pickering high internal phase emulsions was fabricated for the selective binding of $\mathrm{Li}^{+}$. PHIPEs-SS-Ag possessed porous polymer matrix, sufficient sulfonic acid functional groups, and uniformly immobilized silver particles, which were beneficial for improving mass transfer, binding amount and antifouling performance. In batch mode experiments, the adsorption capacity reached a maximum value (i.e. $14.09 \mathrm{mg} \mathrm{g}^{-1}$ ) under alkaline conditions, and the adsorption mechanism between PHIPEs-SS-Ag and $\mathrm{Li}^{+}$ was electrostatic attraction. PHIPEs-SS-Ag exhibited fast binding kinetics at $25^{\circ} \mathrm{C}$ (i.e. $300 \mathrm{~min}$ ), and the maximum monolayer adsorption amount from the Langmuir model for $\mathrm{Li}^{+}$are $59.85 \mathrm{mg} \mathrm{g}^{-1}, 35.06 \mathrm{mg}$ $\mathrm{g}^{-1}$, and $27.09 \mathrm{mg} \mathrm{g}^{-1}$ at $15{ }^{\circ} \mathrm{C}, 25^{\circ} \mathrm{C}$, and $35{ }^{\circ} \mathrm{C}$, respectively. Moreover, PHIPEs-SS-Ag displayed excellent selectivity for $\mathrm{Li}^{+}$in the presence of $\mathrm{K}^{+}, \mathrm{Mg}^{2+}$, and $\mathrm{Na}^{+}$, and maintained $80.71 \%$ of the initial adsorption capacity after seven sequential cycles of adsorption-regeneration. Therefore, this work opened up a universal route for the development of composite adsorbents for the specific separation of $\mathrm{Li}^{+}$.
\end{abstract}

\section{Introduction}

Lithium is widely used in various fields of national economy such as ceramics, glass, lithium-ion batteries, and nuclear energy due to its excellent properties. ${ }^{1}$ The demand for lithium increases year by year, with the continuous expansion of the application field. ${ }^{2,3}$ Therefore, it is necessary to improve the production methods of lithium ions in order to meet the huge market demand. Lithium-rich minerals and salt lakes are the two major sources of lithium. ${ }^{4,5}$ China has a very rich resource of salt lakes, with a lithium content as high as $25 \% .^{5}$ The extraction of lithium ion from salt lakes has the advantages of being low price and environmentally friendly. However, current extraction techniques still have many difficulties, especially because of the presence of interfering ions including $\mathrm{K}^{+}, \mathrm{Na}^{+}$, and $\mathrm{Mg}^{2+}{ }^{6,7}$ Until now, enormous methods such as calcination, precipitation and solvent extraction have already been used to improve the extraction of lithium. ${ }^{8}$ Unfortunately, the calcination method causes serious corrosion to the equipment, which may cause environmental pollution. ${ }^{9}$ Moreover, the high price of the precipitant used in the precipitation method leads to the poor prospect of industrial application. ${ }^{\mathbf{1 0}}$ Furthermore, a large

${ }^{a}$ School of Environmental and Chemical Engineering, Jiangsu University of Science and Technology, Zhenjiang, Jiangsu 212003, China. E-mail: pyxhx@just.edu.cn; Fax: +86 88791800; Tel: +8688791708

${ }^{b}$ School of Chemistry and Chemical Engineering, Jiangsu University, Zhenjiang 212013, China.E-mail:pjm@ujs.edu.cn amount of organic waste liquid is easily produced by solvent extraction. ${ }^{11}$

A fantastic technique available is called adsorption separation, ${ }^{\mathbf{1 2 - 1 4}}$ which shows promise in this regard due to the advantages of its low cost, high efficiency, and easy processing and recycling ability. It has been successfully used for gas separation, air purification, and wastewater treatment. ${ }^{\mathbf{1 5}} \mathrm{S}$. Choudhury $^{\mathbf{1 6}}$ et al. reported a lithium-adsorption material with low efficiency due to the long mass diffusion path length. High internal phase emulsions (HIPEs), in which the internal phase volume fraction exceeds $74 \%$, are the novel templates for the fabrication of porous polymers. ${ }^{17-19}$ In the case of using colloidal particles as a stabilizer or colloidal particles, the formed emulsions are referred to as Pickering HIPEs. ${ }^{20,21}$ Porous polymers derived from HIPEs are potential adsorbents because they are capable of improving mass transfer and the efficiency of adsorption. In addition, the introduction of stabilizer particles could enhance mechanical performance. For example, a porous material with a highly interconnected pore network derived from a Pickering HIPEs template was reported to effectively bind copper ions. ${ }^{22}$

Recently, Chen's group proposed an important work about metal-organic frameworks (MOFs) modified with sulfonic acid groups for the extraction of lithium ions. ${ }^{23}$ In this work, sulfonic acid groups were considered as the available binding agent for lithium ions. However, the introduction of sulfonic acid groups into porous material via HIPEs has not been reported. To date, biofouling, the process by which organisms attach to 
underwater adsorbents, poses a major economic burden for separation industries. Fouling organisms that settle on submerged surfaces increase hydrodynamic drag, lower binding sites, and, in turn, increase non-specific adsorption. ${ }^{24,25}$ Silver is an effective antibacterial agent, which has been immobilized on different support materials for a wide range of antimicrobial and antifouling performance applications. ${ }^{26}$ To the best of our knowledge, using Pickering HIPEs to produce the nano-silver immobilized porous material was rarely reported.

In this work, halloysite nanotubes (HNTs), ${ }^{27}$ a very promising nanomaterial because of its hollow tubular morphology, large specific area, and tunable surface chemistry, was first considered as a stabilizer to form oil-in-water O/W Pickering HIPEs. Then, porous polystyrene sulfonate derived from Pickering HIPEs (PHIPEs-SS) was simply prepared, and sodium $p$-styrene sulfonate (SS) in the external phase was adopted as the functional monomer. Finally, silver-modified porous polystyrene sulfonate (PHIPEs-SS-Ag) was fabricated via an in situ approach in the presence of $\mathrm{NaBH}_{4}$ solution. The adsorption kinetics, equilibrium, selectivity and regeneration of PHIPEs-SS-Ag towards lithium ions were thus investigated, and the antimicrobial performance was also discussed.

\section{Experimental}

\subsection{Materials}

Halloysite nanotubes (HNTs) were obtained from Zhengzhou Jinyangguang Chinaware Co., Ltd. (Henan, China). Tween-80, acrylamide (AM), $N, N^{\prime}$-methylenebisacrylamide (BAM, 99\%), sodium $p$-styrene sulfonate (SS, $90 \%)$, and silver nitrate $\left(\mathrm{AgNO}_{3}\right.$, 99.0\%) were supplied by Aladdin Reagent Co., Ltd. (Shanghai, China). Ammonium persulfate (APS, 98\%), liquid paraffin, $\mathrm{LiCl} \cdot \mathrm{H}_{2} \mathrm{O}$, ethanol, acetone, sodium chloride $(\mathrm{NaCl})$, and sodium borohydride $\left(\mathrm{NaBH}_{4}\right)$ were purchased from Sinopharm Chemical Reagent Co., Ltd. (Shanghai, China). In addition, peptone, yeast extract, and agar were purchased from SigmaAldrich (Germany). E. coli (ACCN, KJ880039) strains were used for antibacterial experiments. The deionized water used in the experiment was filtered with an ultrapure water instrument, and all chemicals were used without further purification.

\subsection{Characterization}

The images of the Pickering HIPES droplets were obtained via optical microscopy (OM, Shanghai Peter EM (BM) Optical Instrument Manufacturing Company Limited, China). The morphologies of PHIPEs-SS and PHIPEs-SS-Ag were observed by field emission scanning electron microscopy (SEM, JSM-7100F; JEOL Ltd., Japan). Energy dispersive spectrometry (EDS) data were examined through an SEM instrument. Infrared spectra (4000-400 $\mathrm{cm}^{-1}$ ) were performed based on Fourier transform infrared spectrometry (FTIR, NEXUS-470; Nicolet, USA). The curves of the TGA analysis were taken by an integrated thermal analyzer (TGA, Perkin-Elmer, U.S.A.) under a nitrogen atmosphere ranging from $25{ }^{\circ} \mathrm{C}$ to $800{ }^{\circ} \mathrm{C}$ at a heating rate of $5.0^{\circ} \mathrm{C} \mathrm{min}^{-1}$. The specific surface area was determined by the
Brunauer-Emmett-Teller method (BET, ASAP 2420 HD88, Micromeritics, U.S.A.). The elemental analysis of two polymer materials was detected via an element analyzer (EL, FLASH112A; Thermo Electron Ltd., American). XPS spectra of PHIPEs-SS and PHIPEs-SS-Ag were recorded through X-ray photoelectron spectroscopy (XPS, ESCALAB 250; Thermo Electron Ltd., American). The concentration of lithium ions was measured by inductively coupled plasma emission spectrometry (ICP, VISTA-MPX; Varian Australia Ltd., Australia).

\subsection{Preparation of porous polystyrene sulfonate derived from Pickering HIPEs (PHIPEs-SS)}

The fabrication of PHIPEs-SS was carried out by an emulsiontemplated method. Firstly, a uniform aqueous phase was prepared by dispersing $0.08 \mathrm{~g}$ of HNTs and $0.04 \mathrm{~g}$ of Tween-80 into $4.0 \mathrm{~mL}$ of deionized water, after which the mixture was sonicated for $30 \mathrm{~min}$. Then, $0.7 \mathrm{~g}$ of AM, $2.5 \mathrm{~g}$ of SS, $0.309 \mathrm{~g}$ of BAM, and $0.02 \mathrm{~g}$ of APS were continuously injected to the aqueous phase (i.e. external phase), and this mixture was continuously stirred for $1.0 \mathrm{~h}$ (Scheme 1A). To obtain stabilized $\mathrm{O} / \mathrm{W}$ Pickering HIPEs, $16 \mathrm{~mL}$ of liquid paraffin as the oil phase (i.e. internal phase) was added into the mixed aqueous phase dropwise under continuous stirring for $3.0 \mathrm{~h}$. Next, the asprepared emulsion was moved to a $25 \mathrm{~mL}$ ampere bottle and polymerized at $70{ }^{\circ} \mathrm{C}$ for $24 \mathrm{~h}$. Afterward, the product (i.e. PHIPEs-SS) was oven-dried at $50{ }^{\circ} \mathrm{C}$ (Fig. 1) overnight in a vacuum oven until it reached a steady weight. Finally, PHIPEsSS as washed with acetone via Soxhlet extraction to extract the internal oil phase and small molecules. In order to obtain the optimized amount of SS, $2.5 \mathrm{~g}(0.012 \mathrm{~mol})$ and $3.0 \mathrm{~g}(0.015 \mathrm{~mol})$ of SS were used to prepare PHIPEs-SS, and the products were named as PHIPEs-0.012SS and PHIPEs-0.015SS, respectively.

\subsection{Synthesis of silver-modified porous polystyrene sulfonate (PHIPEs-SS-Ag)}

An in situ approach was used to fabricate PHIPEs-SS-Ag. $10 \mathrm{mg}$ of PHIPEs-SS was soaked in a centrifuge tube containing $10 \mathrm{~mL}$ of $\mathrm{AgNO}_{3}(1.36 \mathrm{mg}, 8.0 \mu \mathrm{mol})$ solution. Subsequently, the centrifuge tube was immediately sealed after nitrogen purging and was then shaken gently. After $24 \mathrm{~h}$, the soaked foams were taken out and placed directly into boiling aqueous $\mathrm{NaBH}_{4}$ solution $(24.2 \mathrm{mg}, 640 \mu \mathrm{mol}, 20 \mathrm{~mL}$ ) for $15 \mathrm{~min}$ (Scheme 1A). Finally, silver-modified porous polystyrene sulfonate (PHIPEsSS-Ag) was washed with deionized $\mathrm{H}_{2} \mathrm{O}$ and dried in a vacuum oven at about $70{ }^{\circ} \mathrm{C}^{24}$ The products using PHIPEs-0.012SS and PHIPEs-0.015SS were named as PHIPEs-0.012SS-Ag and PHIPEs$0.015 \mathrm{SS}-\mathrm{Ag}$, respectively.

\subsection{Antimicrobial activity}

The antimicrobial activity of PHIPEs-SS and PHIPEs-SS-Ag were detected against the model E. coli bacterial strains by forming a zone of inhibition. The LB (Luria-Bertani) broth ${ }^{28}$ contained $1.0 \mathrm{~g}$ of peptone, $0.5 \mathrm{~g}$ of yeast extract, $0.5 \mathrm{~g}$ of sodium chloride, $3.0 \mathrm{~g}$ of agar, and $100 \mathrm{~mL}$ of water. $40-50 \mu \mathrm{L}$ of bacteria was added to $4.0 \mathrm{~mL}$ of the nutrient solution above, and the bacteria were cultured by shaking for $15-20 \mathrm{~h}$. Then, $1.0 \mathrm{~mL}$ of diluted 
A

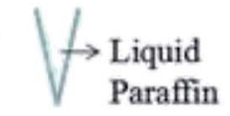
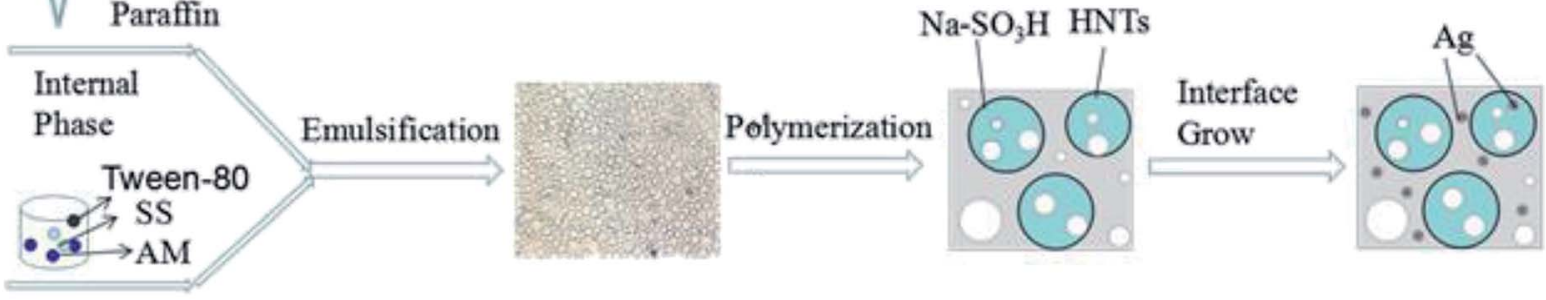

\section{External \\ Phase}

B

$\mathrm{Li}^{+}$
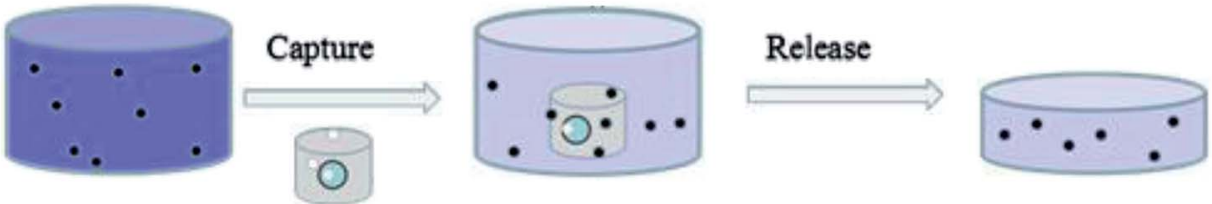

Bacteria

Antifouling

Scheme 1 Schematic for the preparation procedure of PHIPEs-SS-Ag (A) and its adsorptive behavior towards $\mathrm{Li}^{+}(\mathrm{B})$.

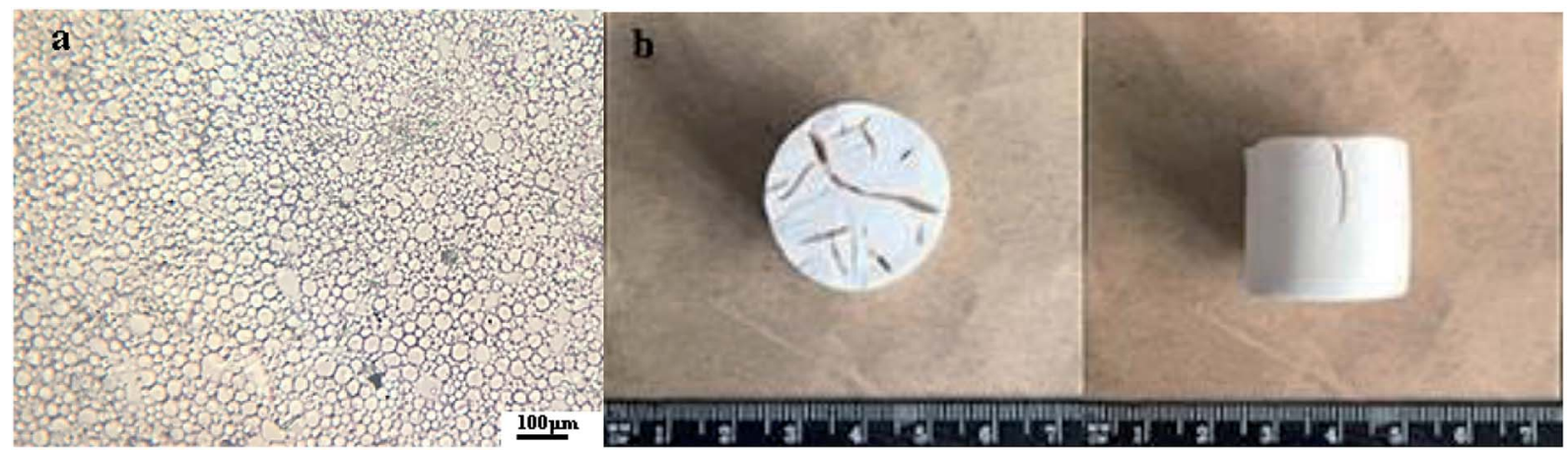

Fig. 1 Micrograph of the Pickering O/W HIPEs-SS (a); PHIPEs-SS monolith (b).

bacteria and $20 \mathrm{~mL}$ of nutrient solution were transferred into the Petri dish, and the mixture was shaken evenly until the liquid showed micro-coagulation. When the cell number reached $10^{8} \mathrm{CFU}$, a piece of filter paper $(2.0 \mathrm{~cm})$, which was soaked in the material (i.e. PHIPEs-SS and PHIPEs-SS-Ag) dispersion $\left(10 \mathrm{mg} \mathrm{mL}^{-1}\right)$, was placed onto the Petri dish, and this plate was allowed to grow for $24 \mathrm{~h}$ at $37^{\circ} \mathrm{C}^{29}$ All pharmaceutical tools were sterilized by autoclave and operated at the beginning of a sterile bench near the flame.

\subsection{Static adsorption experiments}

In the static adsorption experiments, adsorption kinetics and the equilibrium isotherm were considered, and factors such as contact time and the initial concentration of $\mathrm{Li}^{+}$upon adsorption were studied. In the kinetic experiments, PHIPEs-SS-Ag $(10 \mathrm{mg})$ was placed in a $10 \mathrm{~mL}$ centrifuge tube in the presence of $10 \mathrm{~mL}$ of $\mathrm{Li}^{+}$solution with an initial concentration of $150 \mathrm{mg} \mathrm{L}^{-1}$ in a water bath $(298 \mathrm{~K})$. After the desired contact time (10 $\mathrm{min}$ to $720 \mathrm{~min}$ ), PHIPEs-SS-Ag was collected by high-speed centrifugation, and the $\mathrm{Li}^{+}$concentration in the supernatant was detected by inductively coupled plasma emission spectrometry (ICP-ES). ${ }^{30}$ The number of adsorbates adsorbed at time $t\left(Q_{t}, \mathrm{mg} \mathrm{g}^{-1}\right)$ was calculated using the following equation, eqn (1):

$$
Q_{t}=\frac{\left(C_{0}-C_{t}\right) V}{M}
$$

where $C_{0}\left(\mathrm{mg} \mathrm{L}^{-1}\right)$ represented the initial concentrations of $\mathrm{Li}^{+}$, $C_{t}\left(\mathrm{mg} \mathrm{L}^{-1}\right)$ is defined as the residual concentration in the solution at time $t, C_{\mathrm{e}}\left(\mathrm{mg} \mathrm{L}^{-1}\right)$ is the equilibrium concentrations of adsorbates in the solution, $V$ is the volume of the solution $(\mathrm{mL})$, and $M$ is the mass of PHIPEs-SS-Ag $(\mathrm{mg})$. 
To study the adsorption isotherm, a range of initial concentrations $\left(50 \mathrm{mg} \mathrm{L}^{-1}\right.$ to $400 \mathrm{mg} \mathrm{L}^{-1}$ ) of $\mathrm{Li}^{+}$were mixed with $10 \mathrm{mg}$ of PHIPEs-SS-Ag in centrifuge tubes at $288 \mathrm{~K}, 298 \mathrm{~K}$ and $308 \mathrm{~K}$, respectively. After incubation for $6.0 \mathrm{~h}$, the amount of $\mathrm{Li}^{+}$ in the filtrate was determined by ICP-ES. The equilibrium binding amount $Q_{\mathrm{e}}\left(\mathrm{mg} \mathrm{g}^{-1}\right)$ was also calculated by eqn (1), but $C_{t}$ was replaced by the equilibrium concentration $\left(C_{\mathrm{e}}, \mathrm{mg} \mathrm{L}^{-1}\right)$.

\subsection{Binding specificity and regeneration experiments}

In order to measure the specificity of PHIPEs-SS-Ag, $\mathrm{K}^{+}, \mathrm{Mg}^{2+}$, and $\mathrm{Na}^{+}$were selected to be compared with $\mathrm{Li}^{+}$under the same batch conditions. In specific binding experiments, $10 \mathrm{mg}$ of PHIPEs-SS-Ag was added to $10 \mathrm{~mL}$ of single adsorbate solutions of $\mathrm{Li}^{+}, \mathrm{K}^{+}, \mathrm{Mg}^{2+}$, and $\mathrm{Na}^{+}\left(25 \mathrm{mg} \mathrm{L}{ }^{-1}\right)$ at $25{ }^{\circ} \mathrm{C}$, respectively. After incubating for $6.0 \mathrm{~h}$, PHIPEs-SS-Ag was collected by centrifugation, and the concentrations of $\mathrm{Li}^{+}, \mathrm{K}^{+}, \mathrm{Mg}^{2+}$, and $\mathrm{Na}^{+}$(ref. 31) in the filtrate were detected by ICP-ES.

In regeneration experiments, the binding amounts of PHIPEs-SS-Ag were analyzed after seven adsorption-desorption cycles. PHIPEs-SS-Ag was firstly dispersed in $\mathrm{Li}^{+}$solution with a concentration of $150 \mathrm{mg} \mathrm{L}^{-1}$ for $6.0 \mathrm{~h}$ at $25^{\circ} \mathrm{C}$, after which the binding amount was calculated. Then, the collected samples were washed with $0.5 \mathrm{M}$ hydrochloric acid to remove captured $\mathrm{Li}^{+}$.

\section{Results and discussion}

\subsection{Preparation of Pickering O/W HIPEs}

The preparation procedure for PHIPEs-SS-Ag was illustrated in Scheme 1A. Firstly, Pickering O/W HIPEs with an internal-phase volume fraction of $80 \%$ were formed adopting HNTs and few Tween-80 as the emulsifier, in which the aqueous phase was the external phase and the oil phase was the internal phase. The water phase consisted of AM, SS, BAM, APS, and $4.0 \mathrm{~mL}$ of $\mathrm{H}_{2} \mathrm{O}$, and the oil phase contained $16 \mathrm{~mL}$ of liquid paraffin. Secondly, after curing at high temperature, light PHIPEs-SS foams were fabricated. ${ }^{32}$ Finally, silver nanoparticles were modified onto the surface of macroporous monolith via the reaction between immobilized $\mathrm{Ag}^{+}$and $\mathrm{NaBH}_{4} \cdot{ }^{33,34}$ As-prepared PHIPEs-SS-Ag possessed the merits of porous structure and abundant sulfonic acid groups, which were beneficial for the rapid and effective capture of $\mathrm{Li}^{+}$.

\subsection{Characterizations of PHIPEs-SS-Ag}

Fig. 1 showed the images of the Pickering HIPEs-SS droplets (a) and Pickering HIPEs-SS (b). Fig. 1a showed that the emulsion droplets were uniform without obvious demulsification (the droplet microscope images taken after 12 hours of placement), which is sufficient to prove the stability of the emulsion. The substance Pickering HIPEs-SS before curing has a high viscosity, but after curing, it possesses a light foam column structure, as shown in Fig. $1 \mathrm{~b}$.

Fig. 2 showed the SEM images of PHIPEs-0.012SS (a1 and a2), PHIPEs-0.012SS-Ag (b1 and b2), PHIPEs-0.015SS (c1 and c2), and PHIPEs-0.015SS-Ag (d1 and d2), respectively. Four samples all possessed an open-cell structure with interconnecting pores as expected, and the high porosity endowed available permeability and low density. ${ }^{35}$ PHIPEs-0.015SS had many more interconnecting pores and open-cells than those of PHIPEs0.012SS, but the pore throat size of PHIPEs-0.015SS was smaller than those of PHIPEs-0.012SS. This was possibly because the internal phase volume fraction slightly decreased by raising the monomer amount of SS in the aqueous phase, and the smaller droplets were formed during the polymerization process. Moreover, it could be clearly seen that the surfaces of PHIPEs-0.012SS and PHIPEs-0.015SS were smooth, while the surface of PHIPEs-0.012SS-Ag and PHIPEs-0.015SS-Ag were rough, suggesting that the silver particles were immobilized on the cavity surface of the macroporous adsorbents. In addition, after the reaction between $\mathrm{Ag}^{+}$and $\mathrm{NaBH}_{4}$, PHIPEs-SS-Ag retained the foam structure of PHIPEs-SS, indicating that this mild reaction condition had no negative effect on the porous morphology of the material. When compared with PHIPEs$0.015 \mathrm{SS}-\mathrm{Ag}$, the inferior mechanical characterization of
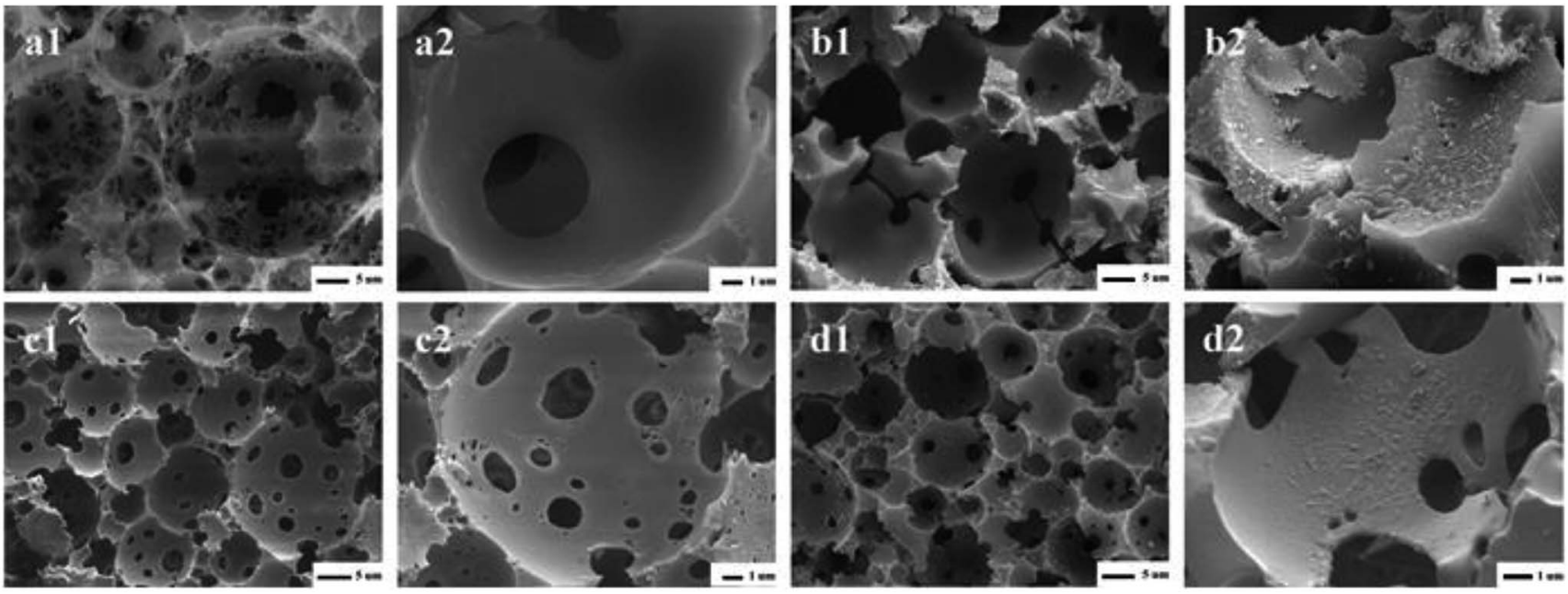

Fig. 2 SEM images of PHIPEs-0.012SS (a1 and a2), PHIPEs-0.012SS-Ag (b1 and b2), PHIPEs-0.015SS (c1) and Area (c2), PHIPEs-0.015SS-Ag (d1) and Area (d2), respectively. 
PHIPEs-0.012SS-Ag was also observed, and this frangible nature could be attributed to the damaged polymer walls. Thus, PHIPEs-0.015SS-Ag was selected as the research object, namely PHIPEs-SS-Ag for subsequent studies.

Fig. 3 shows the half pore size distribution of PHIPEs-SS (a) and PHIPEs-SS-Ag (b) from the $\mathrm{N}_{2}$ adsorption/desorption isotherms as well as the interconnecting pore size (c) and void pore size (d) from the SEM images. ${ }^{36}$ The surface area of PHIPEs-SS and PHIPEs-SS-Ag as determined from the Brunauer-Emmett-Teller (BET) adsorption method were $15.52 \mathrm{~m}^{2}$ $\mathrm{g}^{-1}$ and $16.55 \mathrm{~m}^{2} \mathrm{~g}^{-1}$. The slight increase in the surface area arose from the stacking of immobilized $\mathrm{Ag}$ nanoparticles. As shown in Fig. 3a and b, there were no obvious differences in the half pore size distribution of PHIPEs-SS and PHIPEs-SS-Ag; however, the average half pore size distribution of PHIPEs-SS$\mathrm{Ag}$ slightly decreased from $16.56 \mathrm{~nm}$ to $13.77 \mathrm{~nm}$. This was possibly because few $\mathrm{Ag}$ nanoparticles blocked the pores of the foam structure. The interconnecting pore and void pore sizes of PHIPEs-SS nearly experienced no change, as determined from the SEM images, when compared with PHIPEs-SS-Ag (Fig. 3c and $\mathrm{d}$ ).

The energy dispersive spectrometer (EDS) analysis for PHIPEs-SS and PHIPEs-SS-Ag were listed in Fig. $4 \mathrm{a}$ and $4 \mathrm{~b}$, respectively. As shown in Fig. 4a, the peaks of the elements $\mathrm{Si}$ and $\mathrm{Al}$ indicated that HNTs were attached as stabilized particles. Also, the peak of the element S was obviously displayed, which suggested that $-\mathrm{SO}_{3} \mathrm{H}$ groups from the $\mathrm{SS}$ monomer were also introduced via the polymerization reaction. When compared with Fig. 4a, the peak of the element Ag appeared in PHIPEs-SS-Ag (Fig. 4b), confirming the existence of Ag. The results of elemental analysis were also shown in the inset figures. The amount of S in PHIPEs-SS-Ag slightly decreased by $0.44 \%$ after the immobilization of the $\mathrm{Ag}$ particles. The results demonstrated the mild condition between immobilized $\mathrm{Ag}^{+}$and $\mathrm{NaBH}_{4}$.

FT-IR spectra (a), the X-ray photoelectron spectroscopy spectrum of the survey (b), and the C1s of PHIPEs-SS (c1) and PHIPEs-SS-Ag (c2) were observed in Fig. 5. FT-IR analysis was carried out to verify the composition of PHIPEs-SS and PHIPEsSS-Ag. The absorption bands at $3400 \mathrm{~cm}^{-1}$ and $1650 \mathrm{~cm}^{-1}$ corresponded to the $-\mathrm{NH}_{2}$ and $\mathrm{C}=\mathrm{O}$ stretching vibrations from the AM monomer. The characteristic bands of $1440 \mathrm{~cm}^{-1}$ and $1120 \mathrm{~cm}^{-1}$ were the signals of the benzene ring and $-\mathrm{SO}_{3} \mathrm{H}$ groups from the SS monomer, ${ }^{\mathbf{3 1}, 37}$ respectively. The peak ascribed to the $\mathrm{C}-\mathrm{H}$ bond was observed around $870 \mathrm{~cm}^{-1}$. The band around $1040 \mathrm{~cm}^{-1}$ associated with the $\mathrm{O}-\mathrm{H}$ bending vibration, confirming the presence of HNTs. For PHIPEs-SS-Ag, an additional vibrational band around $1180 \mathrm{~cm}^{-1}$ could be assigned to the Ag, and this result was also confirmed by EDS analysis.

X-ray photoelectron spectroscopy (XPS) spectra of PHIPEs-SS and PHIPEs-SS-Ag were shown in Fig. 5b. The binding energies
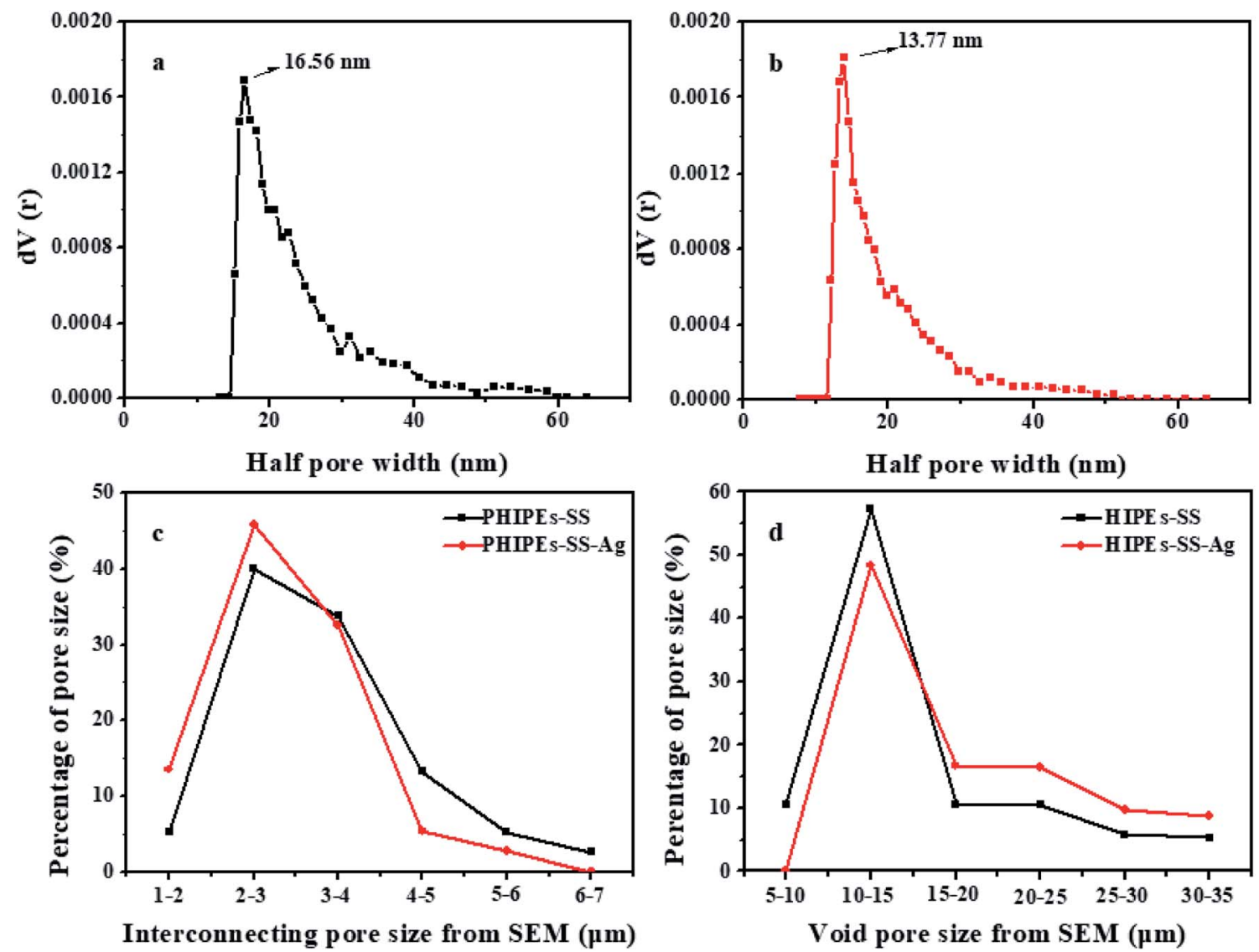

Fig. 3 Half pore size distribution curves in the $\mathrm{N}_{2}$-sorption isotherm of PHIPEs-SS (a) and PHIPEs-SS-Ag (b); interconnecting pore size from SEM (c) and void pore size from SEM (d). 


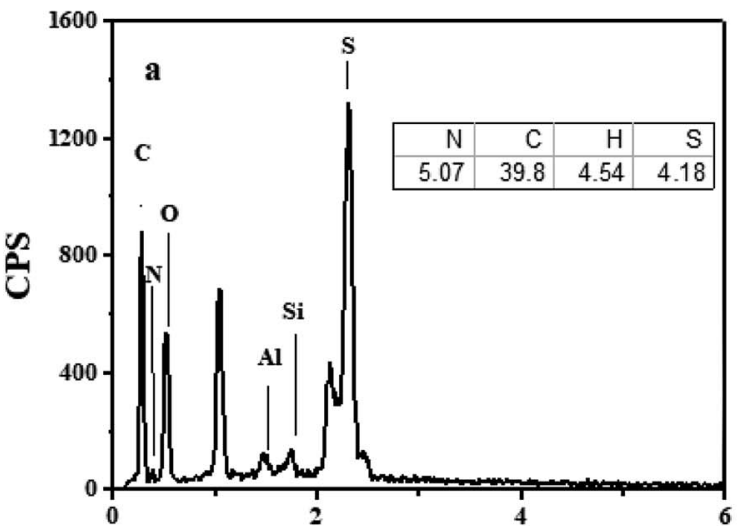

$\mathrm{KeV}$

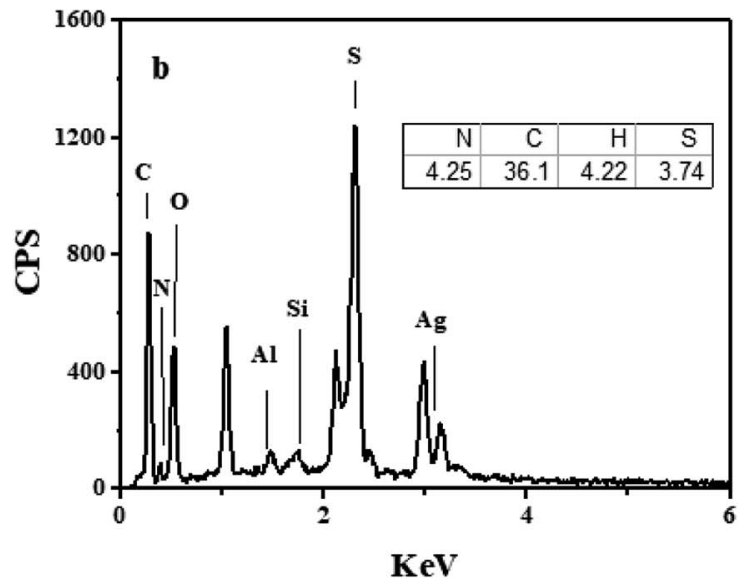

Fig. 4 Energy dispersive spectrometry (EDS) and elemental analysis of PHIPEs-SS (a) and PHIPEs-SS-Ag (b).
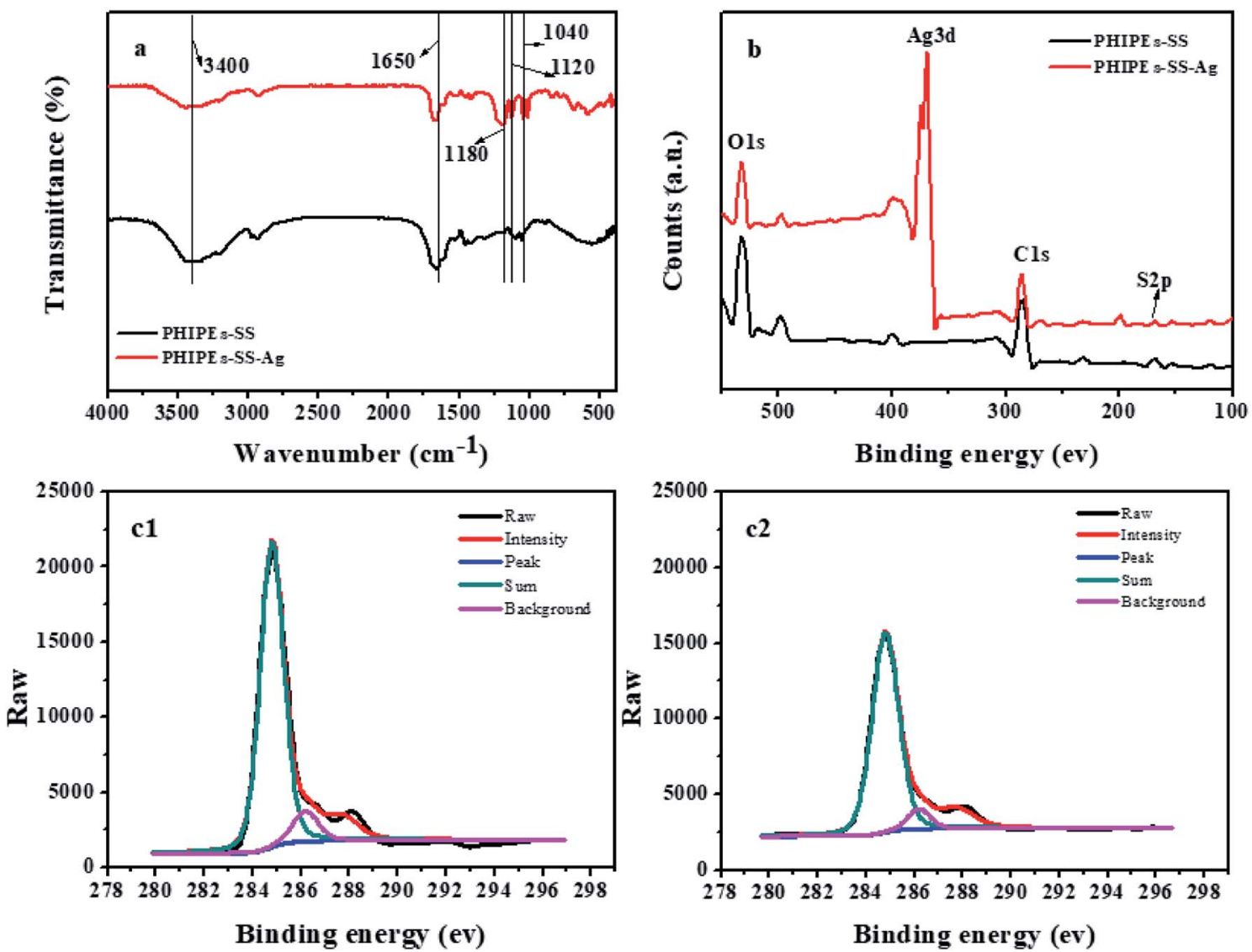

Fig. 5 FT-IR spectra (a); X-ray photoelectron spectroscopy spectrums of survey (b); C1s of PHIPEs-SS (c1) and PHIPEs-SS-Ag (c2).

appearing at $531.83 \mathrm{eV}, 284.79 \mathrm{eV}$, and $168.1 \mathrm{eV}$ can be attributed to the $\mathrm{O} 1 \mathrm{~s}, \mathrm{C} 1 \mathrm{~s}$, and $\mathrm{S} 2 \mathrm{p}$ electrons, respectively, suggesting the presence of $-\mathrm{SO}_{3} \mathrm{H}$ groups. Accordingly, the high-resolution XPS spectrum of C1s (Fig. 5c) showed signals around $284.6 \mathrm{eV}$, $286.2 \mathrm{eV}$ and $287.3 \mathrm{eV}$, which were associated with $\mathrm{C}-\mathrm{C}, \mathrm{C}-\mathrm{SO}_{3}{ }^{-}$ and $\mathrm{O}=\mathrm{C}-\mathrm{N}$ bonds, ${ }^{38}$ respectively. These results further revealed that the two monomers were involved in the polymerization reaction and that the $-\mathrm{SO}_{3} \mathrm{H}$ groups were successfully introduced into the network. Furthermore, a new peak located at $168.1 \mathrm{eV}$ could only be found in PHIPEs-SS-Ag, demonstrating that the Ag nanoparticles were facilely attached to PHIPEs-SS.

Fig. 6 displayed the thermogravimetric (TGA) analysis of PHIPEs-SS and PHIPEs-SS-Ag. With the temperature increasing from $20{ }^{\circ} \mathrm{C}$ to $800{ }^{\circ} \mathrm{C}$, there were three weight loss stages. In the first stage (before $100{ }^{\circ} \mathrm{C}$ ), PHIPEs-SS and PHIPEs-SS-Ag both experienced a slight weight loss, which could be caused by the evaporation of water in the materials. During the second stage 


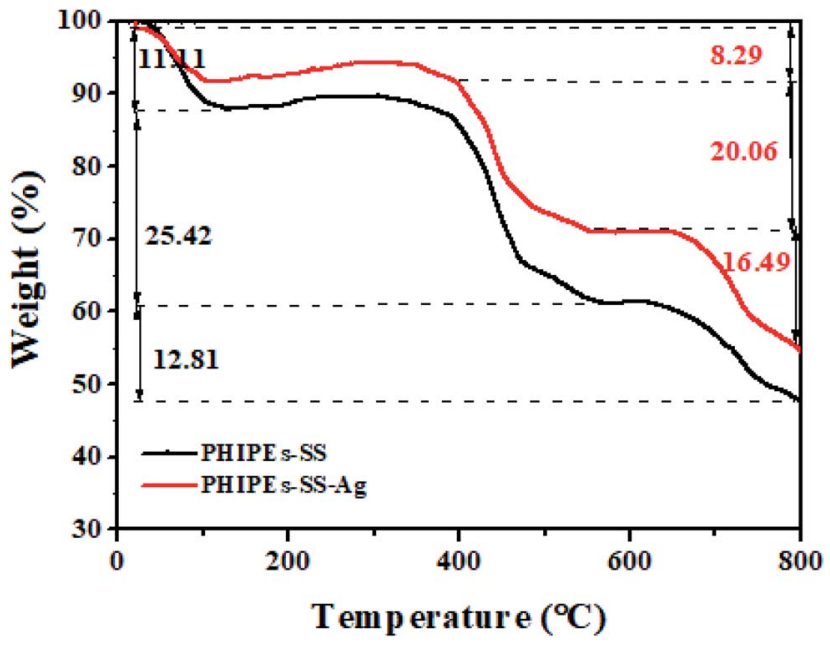

Fig. 6 TGA curves of PHIPEs-SS and PHIPEs-SS-Ag.

$\left(390{ }^{\circ} \mathrm{C}\right.$ to $550{ }^{\circ} \mathrm{C}$ ), the weight loss of PHIPEs-SS and PHIPEs-SS$\mathrm{Ag}$ were $25.42 \%$ and $20.06 \%$, respectively, owing to the thermal decomposition of functional groups, such as the $-\mathrm{SO}_{3} \mathrm{H}$ groups. In the last stage (above $660{ }^{\circ} \mathrm{C}$ ), the conspicuous weight losses may be ascribed to the calcination of the polymer network.

\subsection{Effect of $\mathbf{p H}$}

The effect of $\mathrm{pH}$ on the adsorption capacities of PHIPEs-SS-Ag for $\mathrm{Li}^{+}$at $25{ }^{\circ} \mathrm{C}$ was illustrated in Fig. 7a, and the initial concentrations of $\mathrm{Li}^{+}$was fixed at $150 \mathrm{mg} \mathrm{L}^{-1}$. It was obviously observed that the adsorption capacity of $\mathrm{Li}^{+}$increased along with the increase of solution $\mathrm{pH}^{39}$ eventually reaching a constant value (at a pH ranging from 10 to 11). Additionally, the adsorption capacity attained a maximum value (i.e. $\left.14.09 \mathrm{mg} \mathrm{g}^{-1}\right)$ at $\mathrm{pH}=11$. The zeta potentials were listed in Fig. 7b. The results confirmed the negatively charged surface of PHIPEs-SS-Ag in the tested $\mathrm{pH}$ range, especially under strong alkali conditions. Thus, the adsorption mechanism between PHIPEs-SS-Ag and $\mathrm{Li}^{+}$was electrostatic attraction. Moreover, to avoid possible environmental pollution and the hydrolysis of coexisting metal ions, $\mathrm{pH}=10$ was feasible for effective adsorption in our future work.

\subsection{Adsorption kinetics}

It was evident that the adsorption amount of PHIPEs-0.015SS-Ag was higher than that of PHIPEs-0SS-Ag and PHIPEs-0.012SS-Ag; thus, PHIPEs-0.015SS-Ag (namely PHIPEs-SS-Ag in the following discussion) was selected for adsorption performance studies (Fig. 8a). The kinetic curves for $\mathrm{Li}^{+}$adsorption onto PHIPEs-SS$\mathrm{Ag}\left(\mathrm{pH}=10,25^{\circ} \mathrm{C}\right)$ was shown in Fig. 8b. As shown in Fig. 8b, the adsorption kinetics curve of PHIPEs-SS-Ag could be divided into two processes, a rapid binding during the initial $200 \mathrm{~min}$ and a slow process ranging from $200 \mathrm{~min}$ to $500 \mathrm{~min}$. In the first stage of $\mathrm{Li}^{+}$adsorption, the binding rate was fast and $80.59 \%$ of the equilibrium capacity was obtained due to the abundance of $-\mathrm{SO}_{3} \mathrm{H}$ groups. For the slow stage, the growth of the binding rate was not obvious because of insufficient adsorption sites, and the equilibrium time was observed after $300 \mathrm{~min}$. Moreover, it could be clearly observed that the kinetic curves were continuous and smooth, suggesting the possible fact of the single layer coverage of $\mathrm{Li}^{+}$onto the surface of PHIPEs-SS-Ag. ${ }^{40}$

In order to further study the adsorption kinetics of $\mathrm{Li}^{+}$onto the PHIPEs-SS-Ag surface, the pseudo-first-order kinetic model (PFOKM) and pseudo-second-order kinetic model (PSOKM) were employed to analyze the kinetic results. ${ }^{41}$ The PFOKM and PSOKM were based on the following two equations, which are listed as eqn (2) and (3), respectively:

$$
\begin{array}{r}
Q_{t}=Q_{\mathrm{e}}-Q_{\mathrm{e}} \mathrm{e}^{-k_{1} t} \\
Q_{t}=\frac{k_{2} Q_{\mathrm{e}}{ }^{2} t}{1+k_{2} Q_{\mathrm{e}} t}
\end{array}
$$

where $Q_{t}\left(\mathrm{mg} \mathrm{g}^{-1}\right)$ and $Q_{\mathrm{e}}\left(\mathrm{mg} \mathrm{g}^{-1}\right)$ are the adsorption amounts of $\mathrm{Li}^{+}$by PHIPEs-SS-Ag at time $t$ and at equilibrium, respectively, and $k_{1}\left(\mathrm{~min}^{-1}\right)$ and $k_{2}\left(\mathrm{~g} \mathrm{mg}^{-1} \mathrm{~min}^{-1}\right)$ are the rate constants from the pseudo-first-order and pseudo-second-order models, respectively.

The modeling for the adsorption of $\mathrm{Li}^{+}$onto PHIPEs-SS-Ag was shown in Fig. 8b, and the adsorption rate constants and linear regression values were summarized in Table 1 . The obtained adsorption amount from PSOKM was closer to the experimental data, and the $R^{2}$ value from the two equations also confirmed that $\mathrm{Li}^{+}$adsorption could be well described by PSOKM. This fact could also be proven by the results in Fig. 8b.
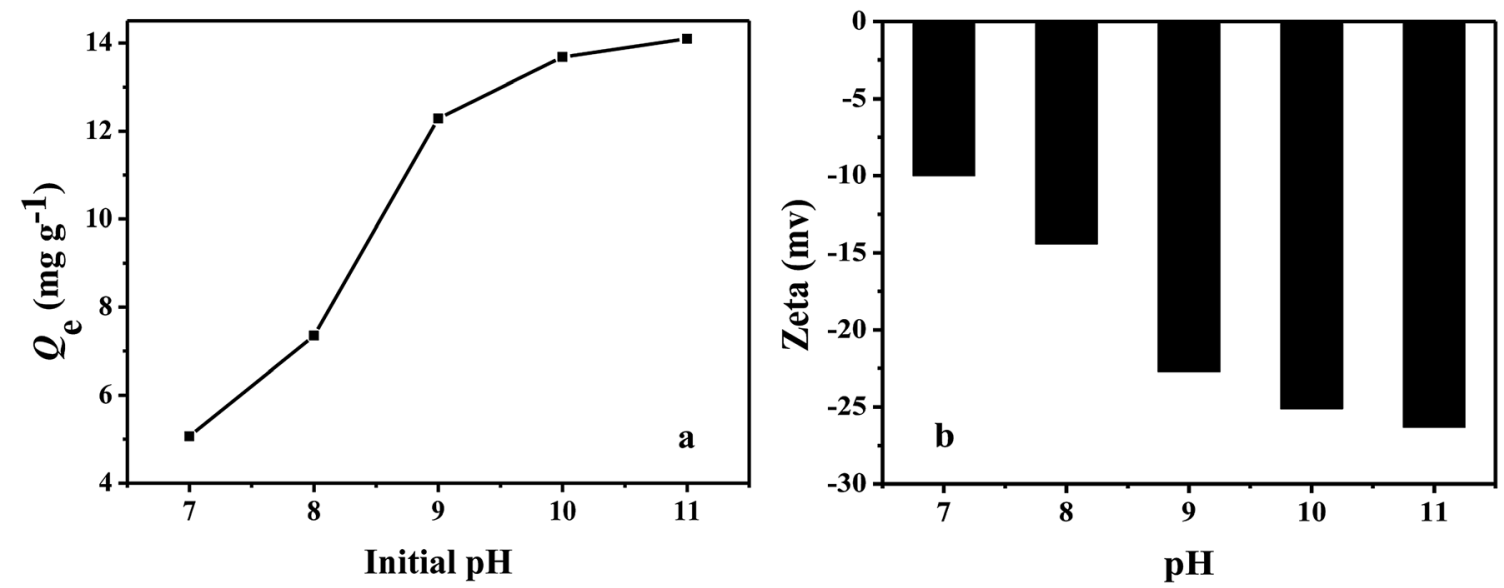

Fig. 7 Effect of $\mathrm{pH}$ on PHIPEs-SS-Ag for the $\mathrm{Li}^{+}$adsorption capacities at $25^{\circ} \mathrm{C}$ (a) and the zeta potential of PHIPEs-SS-Ag at $25{ }^{\circ} \mathrm{C}(\mathrm{b})$. 
Table 2 Adsorption equilibrium constants from Langmuir and Freundlich isotherm equations

\begin{tabular}{lllllll}
\hline & \multicolumn{2}{l}{ Langmuir isotherm model } & & \multicolumn{2}{l}{ Freundlich isotherm model } \\
\cline { 2 - 5 }$T(K)$ & $R^{2}$ & $Q_{\mathrm{m}, \mathrm{L}}\left(\mathrm{mg} \mathrm{g}^{-1}\right)$ & $k_{\mathrm{L}}\left(\mathrm{L} \mathrm{mg}^{-1}\right)$ & & $R^{2}$ & $K_{\mathrm{F}}\left(\mathrm{mg} \mathrm{g}^{-1}\right)$ \\
\hline 288 & 0.9943 & 59.85 & $6.25 \times 10^{-4}$ & 0.9912 & 0.0583 \\
298 & 0.9977 & 35.06 & $1.67 \times 10^{-2}$ & 0.9905 & 0.1608 \\
308 & 0.9982 & 27.09 & $1.90 \times 10^{-2}$ & 0.9900 & 0.1509 \\
\hline
\end{tabular}
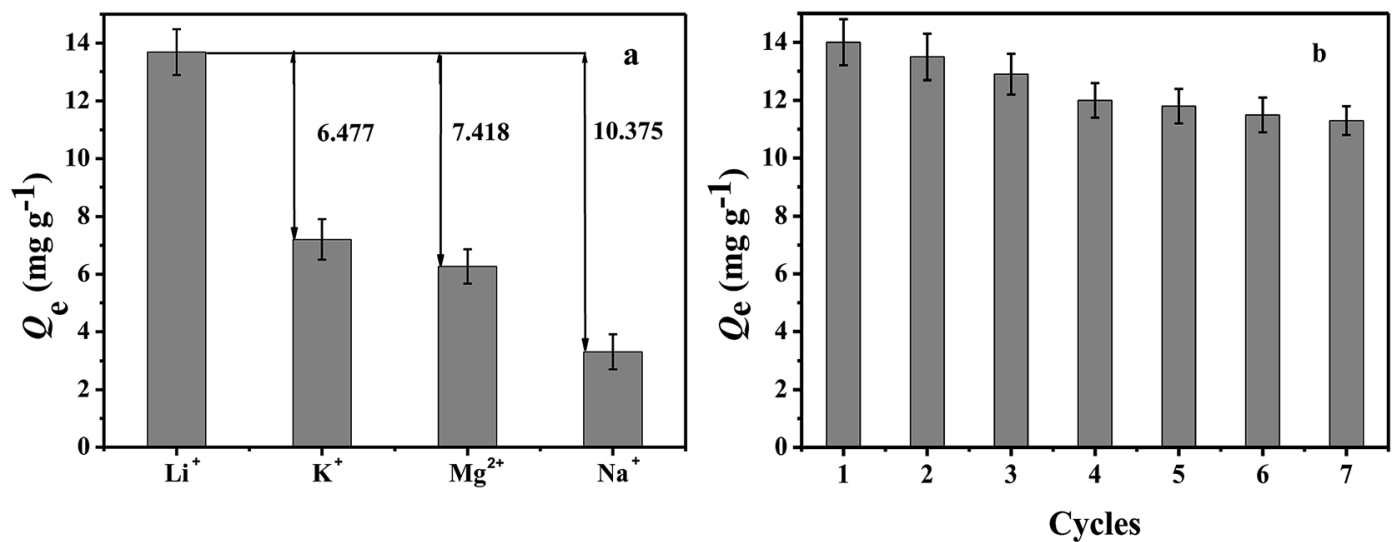

Fig. 10 Adsorption capacity of PHIPEs-SS-Ag for $\mathrm{Li}^{+}, \mathrm{K}^{+}, \mathrm{Mg}^{2+}$, and $\mathrm{Na}^{+}$(a). Adsorption-regeneration capacity of PHIPEs-SS-Ag for $\mathrm{Li}^{+}$removal (initial $\left[\mathrm{Li}^{+}\right]=150 \mathrm{mg} \mathrm{L}^{-1}$ ) (b).

\subsection{Adsorption selectivity, reusability and antibacterial capability}

Adsorption selectivity is an extremely important factor used to evaluate the ability of an adsorbent. In this work, $\mathrm{K}^{+}, \mathrm{Mg}^{2+}$, and $\mathrm{Na}^{+}$were considered as the competitive ions to study the adsorption selectivity of PHIPEs-SS-Ag for $\mathrm{Li}^{+}$. As shown in Fig. 10a, PHIPEs-SS-Ag possessed excellent adsorption ability

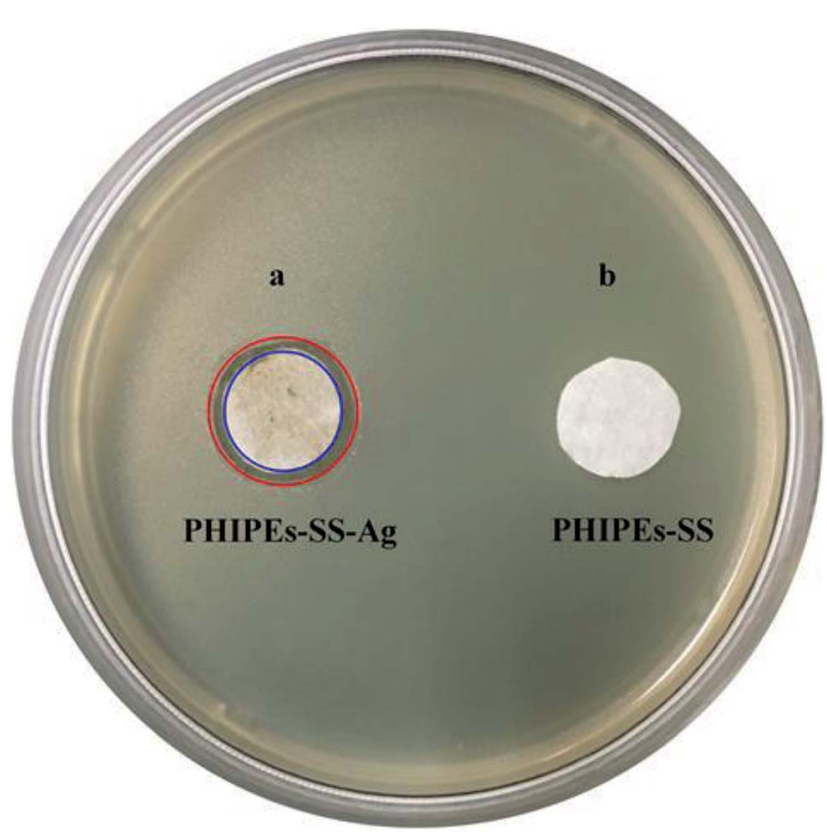

Fig. 11 Inhibition zones of PHIPEs-SS-Ag (a) and PHIPEs-SS (b). for $\mathrm{Li}^{+}$, and the capture amount was superior to that of the other competitive ions. As shown in Fig. 10b, after seven sequential cycles of adsorption-regeneration, the adsorption capacity for $\mathrm{Li}^{+}$remained at $80.71 \%$ of the initial value, owing to the reduction of binding sites in PHIPEs-SS-Ag. Thus, it could be concluded that PHIPEs-SS-Ag had good regeneration ability for the selective capture of $\mathrm{Li}^{+}$. The antimicrobial activity of PHIPEs-SS and PHIPEs-SS-Ag, detecting against the model $E$. coli bacterial strains by forming a zone of inhibition, ${ }^{\mathbf{4 4}}$ suggested that PHIPEs-SS-Ag possessed antibacterial performance, which was beneficial for the antifouling process (Fig. 11).

\section{Conclusions}

In this work, an effective method was demonstrated to fabricate silver-modified porous polystyrene sulfonate (PHIPEs-SS-Ag) via the high internal phase emulsion template and post-synthetic modification. PHIPEs-SS-Ag possessed a highly porous foam structure, an abundance of adsorption sites (i.e., sulfonic acid functional groups), and immobilized Ag nanoparticles. PHIPEsSS-Ag exhibited an excellent adsorption amount, binding kinetics, selectivity, regeneration, and antifouling performance. Our proposed synthesis strategy can be facilely used to prepare functional adsorbents for the selective extraction of $\mathrm{Li}^{+}$.

\section{Conflicts of interest}

There are no conflicts to declare. 


\section{Acknowledgements}

This work was financially supported by the National Natural Science Foundation of China (No. U1607125, 21822807, 21576120, and 21706099), the Natural Science Foundation of Jiangsu Province (No. BK20170491 and BK20170523), the National Postdoctoral Science Foundation of China (No. 2016M600374, and 2017T100341), the Six Talent Peaks Project in Jiangsu Province (No. JNHB-016), and the Zhenjiang Social Development Project (SH2018011).

\section{References}

1 W. Xiang and S. Liang, Hydrometallurgy, 2016, 166, 9-15.

2 C. Grosjean, P. H. Miranda, M. Perrin and P. Poggi, Renewable Sustainable Energy Rev., 2012, 16, 1735-1744.

3 J. M. Tarascon, Philos. Trans. R. Soc., A, 2010, 368, 3227-3241.

4 M. P. Paranthaman, L. Li, J. Q. Luo, T. Hoke, H. Ucar, B. A. Moyer and S. Harrison, Environ. Sci. Technol., 2017, 51, 13481-13486.

5 X. Xu, Y. M. Chen, P. Y. Wan, K. Gasem, K. Y. Wang, T. He, H. Adidharma and M. Fan, Prog. Mater. Sci., 2016, 84, 276313.

6 J. Z. Sun, Y. Dong and C. Y. Kong, Sep. Purif. Technol., 2014, 136, 309-313.

7 A. Somrani, A. H. Hamzaoui and M. Pontie, Desalination, 2013, 317, 184-192.

8 G. M. Nisola, L. A. Limjuco, E. L. Vivas, C. P. Lawagon, M. J. Park, H. K. Shon, N. Mittal, I. W. Nah, H. Kim and W. Chung, Chem. Eng. J., 2015, 280, 536-548.

9 H. Vikström, S. Davidsson and M. Höök, Appl. Energy, 2013, 110, 252-266.

10 Z. W. Zhao, X. F. Si, X. H. Liu, L. H. He and X. X. Liang, Hydrometallurgy, 2013, 133, 75-83.

11 Z. Zhou, W. Qin and W. Fei, J. Chem. Eng. Data, 2011, 56, 3518-3522.

12 S. C. Liu, J. M. Pan, Y. Ma, F. X. Qiu, X. H. Niu, T. Zhang and L. L. Yang, Chem. Eng. J., 2016, 306, 655-666.

13 Q. Jia, Y. Ma, Y. X. Peng, Y. H. Liu and W. L. Zhang, Chem. Eng. J., 2018, 342, 293-303.

14 A. Umeno, Y. Miyai, N. Takagi, R. Chitrakar, K. Sakane and K. Ooi, Ind. Eng. Chem. Res., 2002, 41, 4281-4287.

15 X. C. Zhu, Y. X. Shi, S. Li and N. S. Cai, Int. J. Hydrogen Energy, 2018, 43, 13305-13317.

16 S. Choudhury, D. Connolly and B. White, Anal. Methods, 2015, 7, 6967-6982.

17 J. M. Pan, J. L. Luo, J. Cao, J. X. Liu, W. Huang, W. L. Wen and L. L. Yang, RSC Adv., 2016, 6, 93894-93904.

18 J. Zeng, Y. X. Peng, J. M. Pan, H. P. Gao, R. R. Wu, Y. J. Yin and Y. S. Yan, Chem. Eng. J., 2015, 266, 1-11.

19 J. M. Pan, X. B. Huang, L. Gao, Y. X. Peng, S. C. Liu and R. X. Gu, Chem. Eng. J., 2017, 312, 263-274.

20 Y. Q. He, F. Wu, X. Y. Sun, R. Q. Li, Y. Q. Guo, C. B. Li, L. Zhang, F. B. Xing, W. Wang and J. P. Gao, ACS Appl. Mater. Interfaces, 2013, 5, 4843-4855.
21 H. P. Gao, Y. X. Peng, J. M. Pan, J. Zeng, C. H. Song, Y. L. Zhang, Y. S. Yan and W. D. Shi, RSC Adv., 2014, 4, 43029-43038.

22 J. X. Han and Z. J. Du, J. Hazard. Mater., 2014, 276, 225-231.

23 Y. Guo, Y. L. Ying, Y. Y. Mao, X. S. Peng and B. L. Chen, Angew. Chem., Int. Ed., 2016, 55, 15120-15124.

24 L. Akhigbe, S. Ouki and D. Saroj, Chem. Eng. J., 2016, 295, 9298.

25 S. S. Yuan, S. F. Luan, S. J. Yan, H. C. Shi and J. H. Yin, ACS Appl. Mater. Interfaces, 2015, 7, 19466-19473.

26 M. A. Mudassir, S. Z. Hussain, W. Zaheer, T. M. Ansari and I. Hussain, ACS Appl. Mater. Interfaces, 2017, 28, 2419024197.

27 G. Cavallaro, G. Lazzara, S. Milioto and F. Parisi, J. Am. Chem. Soc., 2015, 31, 7472-7478.

28 S. H. Ko, T. J. Park, H. S. Kim, J. H. Kim and Y. J. Cho, Biosens. Bioelectron., 2009, 24, 2592-2597.

29 R. R. Wu, Y. Ma, J. M. Pan, S. H. Lee, J. X. Liu, H. J. Zhu, R. X. Gu, K. J. Shea and G. Q. Pan, Biosens. Bioelectron., 2018, 101, 52-59.

30 G. R. Zhu, P. Wang, P. F. Qi and C. J. Gao, Chem. Eng. J., 2014, 235, 340-348.

31 Y. Zhao, W. H. Shi, B. V. D. Bruggen, C. J. Gao and J. N. Shen, Adv. Mater. Interfaces, 2018, 5, 1701449.

32 Y. Yang, Z. J. Wei, C. Y. Wang and Z. Tong, Chem. Commun., 2013, 49, 7144-7146.

33 J. Wang, S. S. Zhang, X. Xu, K. X. Fei and Y. X. Peng, Nanomaterials, 2018, 8, 102.

34 J. Wang, K. X. Fei, X. Yang, S. S. Zhang and Y. X. Peng, Materials, 2018, 11, 1291.

35 J. M. Pan, Y. Ma, J. Zeng, X. H. Niu, T. Zhang, F. X. Qiu, Y. X. Peng and Y. S. Yan, Chem. Eng. J., 2016, 302, 670-681.

36 V. O. Ikem, A. Menner, T. S. Horozov and A. Bismarck, Adv. Mater., 2010, 22, 3588-3592.

37 H. P. Gao, J. M. Pan, D. Han, Y. L. Zhang, W. D. Shi, J. Zeng, Y. X. Peng and Y. S. Yan, J. Mater. Chem. A, 2015, 25, 1350713518.

38 S. J. Yan, S. F. Luan, H. C. Shi, X. D. Xu, J. D. Zhang, S. S. Yuan, Y. M. Yang and J. H. Yin, Biomacromolecules, 2016, 17, 1696-1704.

39 X. C. Xu, Y. Li, D. Yang, X. D. Zheng, Y. Y. Wang, J. M. Pan, T. Zhang, J. C. Xu, F. X. Qiu, Y. S. Yan and C. X. Li, J. Cleaner Prod., 2018, 171, 264-274.

40 Q. Jia, Y. X. Peng, J. M. Pan, X. H. Niu and T. Zhang, New J. Chem., 2017, 41, 3308-3319.

41 Y. S. Ho and G. McKay, Process Biochem., 1999, 34, 451-465. 42 I. Langmuir, J. Am. Chem. Soc., 1918, 40, 1361-1403.

43 M. D. LeVan and T. Vermeulen, J. Phys. Chem., 1981, 85, 3247-3250.

44 M. L. Chen, L. P. Ouyang, T. Lu, H. Y. Wang, F. H. Meng, Y. Yang, C. Q. Ning, J. Z. Ma and X. Y. Liu, ACS Appl. Mater. Interfaces, 2017, 9, 16824-16833. 